\title{
The Research of Method Situation Assessment in Robot-Soccer Games Based on Conditional Event Algebra
}

\author{
S. G. Zhu, P. Li, and L. J. Zhan
}

\begin{abstract}
This paper mainly research on the situation assessment method. Since the situation assessment plays a key role in decision-making system, and the correct and effective intelligent decision has a direct decisive effect on winning in football matches. By using the Bayesian network framework to express the relation between events, and combining Conditional Event Algebra for logical reasoning, this paper firstly introduced the basic principle and property about CEA and product space conditional event algebra (PSCEA). And then a new situation assessment method is proposed in situation assessment module to address the incompatible problem between the probability and the logic. The experimental result shows that this new situation assessment method has more intelligent, efficient.
\end{abstract}

Index Terms-Conditional event algebra; Situation assessment.

\section{INTRODUCTION}

As the rapid development of Robot Soccer InTechnology, the damand of robots' intelligence is more and more high. Situation assessment, which plays an important role in making an accurate and intelligent judgment on field, is a assessment processing for the arrangement of robots and the dymanic situation in the field by assessing and analyzing the activity of robots. Up to now, the domestic research departments haven't an agreement in the definition of the Situation assessment. In this paper, we research the Situation Assessment based on Conditional Event Algebra (CEA). CEA has wide application prospects in the field of data fusion, for it can solve the incompatible problem between the probability and the logic. the paper firstly introduced the basic principle and property about CEA and product space conditional event algebra(PSCEA). then proposes method of situation assessment in Robot-Soccer Games based on CEA.

\section{CEA}

\section{A. The Definition of CEA}

Let $(\Omega, B, P)$ be a given probability space, $\Omega$ a sample space, $\mathrm{B}$ a fixed event domain in the space $\Omega$, and $\mathrm{P}$ a certain probability measure. For $s \in \mathrm{B}, p(s)$ defined on the subsets of $S$ belonging to $\sigma$ - algebra $\sigma(\Omega)$ of subsets of

Manuscript received October 20, 2012; Revised November 5, 2012.

Shengguo Zhu and Lijuan Zhan are with Huazhong University of Science \& Technology, Wuhan, China (Email:shengguoz@126.com, Email:276115092@qq.com)

Peng Li is with HuBei Radio and TV University, Wuhan, China (Email: peling9901@163.com).
$\Omega$, that satisfies the following properties[1-3]:

- $p(\Phi)=0, p(\Omega)=1$ 。

- $p\left(s_{1} \cup \cdots \cup s_{x j} \cdots\right)=p\left(s_{1}\right)+\cdots p\left(s_{j}\right)+\cdots$ 。

- The set of all conditional events is a Boolean algebra set with logic connectives $\vee, \wedge$ and '.

- $\left(s \mid \Omega_{0}\right)=s$ 。

- Any probability measure $P$ defined on the events of $\Omega$ can be uniquely extended a probability measure $P_{0}$ on conditional events such that $P_{0}((S \mid X))=p(S \mid X) 。$

- $(S \mid X) \wedge X=(S \mid X)$ 。

\section{B. The definition and properties of PSCEA}

Let $(\Omega, B, P)$ be a given probability space. Given no condition events $a, b, c, d \cdots$, then constructing an extended production probability measurable space $\left(\Omega_{0}, B_{0}, P_{0}\right)[4-5]$

$\Omega_{0}=\Omega \times \Omega \times K, P_{0}$ is probalility measure in the space and $B_{0}$ is a Boolean algebra or $\sigma$ - algebra. So

$$
\begin{aligned}
(a \mid b)=(( & \left.a \wedge b) \times \Omega_{0}\right) \vee\left(\left(b^{\prime} \times a \wedge b\right) \times \Omega_{0}\right) \\
& \vee\left(b^{\prime} \times b^{\prime} \times(a \wedge b) \times \Omega_{0}\right) \\
& \vee\left(b^{\prime} \times b^{\prime} \times b^{\prime} \times(a \wedge b) \times \Omega^{0}\right) \cdots
\end{aligned}
$$

Given a function $f: B \times B \rightarrow B_{0}$, for all $f$ are defined as[5]

$$
\begin{gathered}
f(a, b)=a b \vee\left(b^{\prime} \times a b\right) \vee\left(b^{\prime} \times b^{\prime} \times a b\right) \vee \cdots \\
P_{0}(f(a, b))=P_{0}\left(a b \vee\left(b^{\prime} \times a b\right) \vee\left(b^{\prime} \times b^{\prime} \times a b\right) \vee \cdots\right) \\
=P(a b)+P\left(b^{\prime}\right) P(a b)+P\left(b^{\prime}\right)^{2} P(a b)+\cdots \\
=P(a b) \times \sum_{j=0}^{\infty} P\left(b^{\prime}\right)^{j} \\
=P(a b) \frac{1}{1-P\left(b^{\prime}\right)}=P(a b) \frac{1}{P(b)} \\
=P(a \mid b)
\end{gathered}
$$

According to above definition, In $R$, for any probability 
measure $P, P_{0}((a \mid b))=P(a \mid b) .\left(\Omega_{0}, B_{0}, P_{0}\right)$ extends $(\Omega, B, P)$, and $P_{0}$ extends $P[6]$.

\section{The Method of Situation Assessment Based on CEA}

The higher-order condition event "if if $b_{1}$ then $a_{1}$ and if $b_{2}$ then $a_{2}$ then if $d$ then $c$ "can be expressed as the scheme $^{[7]}$

$$
G=\left[(a \mid b)_{J} ;(c \mid d)\right]
$$

The $a_{J}, b_{J}, c, d$ in Eq.(3) are events under Boolean algebra, $(a \mid b)_{J}$ expresses $\left(a_{j} \mid b_{j}\right)_{j \text { in } J} . P$ is a function denoted as $P: B \rightarrow[0,1] .(a \mid b)_{J}$ is a set of given rules or casual relations, $(c \mid d)$ is inferred conclusion from $(a \mid b)_{J}$.According to CEA, $G$ can be denoted as a set of conjunction events ${ }^{[7]}$ :

$$
\begin{aligned}
A(G)= & \bigcap\left\{a_{j} b_{j}, a_{j}^{\prime} b_{j}, b_{j}^{\prime}\right\} \cap\left\{c d, c^{\prime} d, d^{\prime}\right\} \\
& =\left\{w_{1}, \cdots, w_{m+1}\right\}
\end{aligned}
$$

If no conjunction were null then $m=3^{\operatorname{card}(J)+1}-1, J$ is the collection of all $j$ in $\left(a_{j} \mid b_{j}\right)_{j \text { in } J}, \operatorname{card}(\mathrm{J})$ is dimension of set J. According to Eq. (2), $G=\left[(a \mid b)_{J} ;(c \mid d)\right]$ can be denoted as

$$
f\left(a_{j}, b_{j}, c, d\right)=\vee\left(w_{j}\right), j \text { in } I(f)
$$

Form Eq. (5), for any fuction $f$ in $A(G)$, there exists one and only index set $I(f)$ in $\{1, \cdots, m, m+1\}$. For any probality measure $P, P: B \rightarrow[0,1]$, and

$$
\begin{aligned}
& p\left((a \mid b)_{J} ;(c \mid d)\right)=P(A(G)) \\
& \quad=P_{0}\left(f\left(a_{j}, b_{j}, c, d\right)\right) \\
& \quad=\sum P\left(w_{j}\right), j \text { in } I(f)^{[8]}
\end{aligned}
$$

So, Caculating the value of $G$ is equal to calculate the probility measure of $w_{1}, \cdots, w_{m+1}$ events in tradition probality space. we calculates the value of $P\left(w_{1}\right), \cdots, P\left(w_{m+1}\right)[9]$ and sum up. Then we get the value of $P\left((a \mid b)_{J} ;(c \mid d)\right)$.

Given the scheme $G=\left[(a \mid b)_{J} ;(c \mid d)\right]$ and corresponding Bias network, see from Fig 1, then calculate the value of $p\left((a \mid b)_{J} ;(c \mid d)\right)$. This is the steps of Bayes Logic reasoning algorithm $^{[10-12]}$ based on CEA:

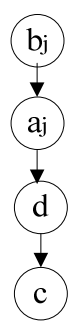

Fig. 1. The structure of Bias network
Step1: The question that we needed to solve is denoted by the higher-order condition event $G=\left[(a \mid b)_{J} ;(c \mid d)\right]$.

Step2: Turn higher-order condition event $G$ into the joins among events $w_{1}, \cdots, w_{m+1}$ by applying the CEA properties.

Step3: Calculate the values of $P\left(w_{1}\right), \cdots, P\left(w_{m+1}\right)$ 。

Step4: sum up $P\left(w_{1}\right), \cdots, P\left(w_{m+1}\right)$ 。

In the algorithm mentioned above, step 3 is excuted as following:

Let $G_{j}=\left[(a \mid b)_{j} ;(c \mid d)\right]$,According to Eq.

(4), $A\left(G_{j}\right)=\left\{a_{j} \times b_{j}, a_{j}^{\prime} \times b_{j}, b_{j}^{\prime}\right\} \cap\left\{c d, c^{\prime} d, d^{\prime}\right\}$

$$
=\left\{w_{1}, \cdots, w_{9}\right\}
$$

So we need to calculate the value of $P\left(w_{i}\right)$ Take calculating the value of $P\left(w_{1}\right)$ for example. $\quad P\left(w_{1}\right)=P\left(a_{j}, b_{j}, c, d\right)$ $=P\left(b_{j}\right) P\left(a_{j} \mid b_{j}\right) P\left(d \mid a_{j} b_{j}\right) P\left(c \mid a_{j} b_{j} d\right)$.

According to the given $a, b, c, d$ and the Bias network probability, we can calculate the values of $P\left(a_{j} \mid b_{j}\right), P\left(d \mid a_{j} b_{j}\right), P\left(c \mid a_{j} b_{j} d\right)$, then calculate the value of $P\left(w_{1}\right)$.

The algorithm firstly induces the problem $P(A(G))$ according to the Bias network topology structure. The problem can be solved in tradition profanity space, for the logic can't be ccompatible with the probability, so turn the higher-order condition event into the joins of normal events by applying the CEA properties, finally calculate the value of $P(A(G))$.

\section{The Application of the Method of Situation ASSESSMENT}

If you are using Word, use either the Microsoft Equation Editor or the MathType add-on (http://www.mathtype.com) for equations in your paper (Insert | Object | Create New | Microsoft Equation or MathType Equation). "Float over text" should not be selected.

The environment of Robot Football Game is dynamic and continuous, so the factors influencing the situation are so many. This paper chooses three most important factors, a is the factor of distance that compare the distance between our team and opposite team to the football. $b$ is the Ball control rate of one team, $c$ is the distance from the ball to opposite team.

$a 1$ is near, $a 2$ is far. $b 1$ is the ball in the control of our team, $b 2$ is the ball in control of the opposite team.c1 is near, $c 2$ is far. $d$ is the game situation of one team,d1 is superiority, $d 2$ is inferiority.

Step 1: certain the Bias network topology structure. We can get Bias network module and the condition probability from the plentiful experience in the games. We can see from Fig. 2.

$$
\begin{aligned}
& \text { Step 2: } G=(d \mid(a \mid b), c), \\
& G=[(a \mid b),(c \mid \Omega) ;(d \mid \Omega)], \text { then } \\
& A(G)=\left\{a b, a^{\prime} b, b^{\prime}\right\} \wedge\left\{c \times \Omega, c^{\prime} \times \Omega\right\} \wedge\left\{d \times \Omega, d^{\prime} \times \Omega\right\} \\
& =\left\{w_{1}, \cdots, w_{12}\right\}=\left\{a b c d, a^{\prime} b c d, b^{\prime} c d, a b c^{\prime} d, a^{\prime} b c^{\prime} d,\right.
\end{aligned}
$$




\section{$b^{\prime} c^{\prime} d, a b c d^{\prime}, a^{\prime} b c d^{\prime}, b^{\prime} c d^{\prime}, a b c^{\prime} d$ ', $\left.a^{\prime} b c^{\prime} d^{\prime}, b^{\prime} c^{\prime} d^{\prime}\right\}$}

Step 3: calculate the value of $P\left(w_{1}\right), \cdots, P\left(w_{12}\right)$. Given the state $a=a 1, b=b 1, c=c 1 \circ$ Assume $d=d 1$,

$$
P\left(w_{1}\right)=p(a b c d)=p(a) p(b \mid a) p(c) p(d \mid b c)
$$$$
=0.6 \times 0.9 \times 0.4 \times 0.8=0.1728 \circ P\left(w_{2}\right), \ldots
$$

$$
P\left(w_{12}\right) \text { are }
$$

$0.0896,0.0648,0.2916,0.1512,0.0756,0.0432,0.0224,0.0072$, $0.0324,0.0168,0.0324$, respectively 。

Step 4: sum up $P\left(w_{1}\right), \cdots, P\left(w_{12}\right)$. The sum is $p(d \mid(a \mid b), c)=0.935$. Because 0.935 approximates to 1 , so the assuming is ture. The result of assessment is superiority.

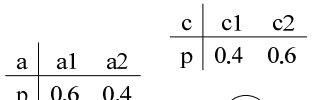

$$
\begin{aligned}
& \begin{array}{c|cc|c|cc}
\hline \mathrm{p} & 0.6 & 0.4
\end{array}
\end{aligned}
$$

Fig. 2. Bias network structure and the condition probability

This is a application example mentioned above, the method of situation assessment is applied to intelligent referee system in the Robot Soccer Simulation platform. According to Fig. 2, Fig. 3.we can see an example in the intelligent referee system. Form Fig. 3, the number 8 teamer in blue team kick the ball to the goal. Form Fig. 4(a), The number 5 teamer in blue team deliberate foul (More than two robots of the defending team in the penalty area (including the goal area)), at that time, the intelligent referee doesn't give any judgment. We can see from Fig. 4(b). This example expresses the intelligence and correctness of the intelligent referee.

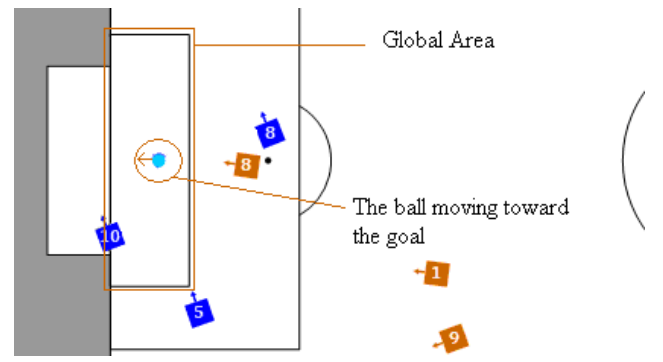

Fig.3. The attack of yellow in the tenth period

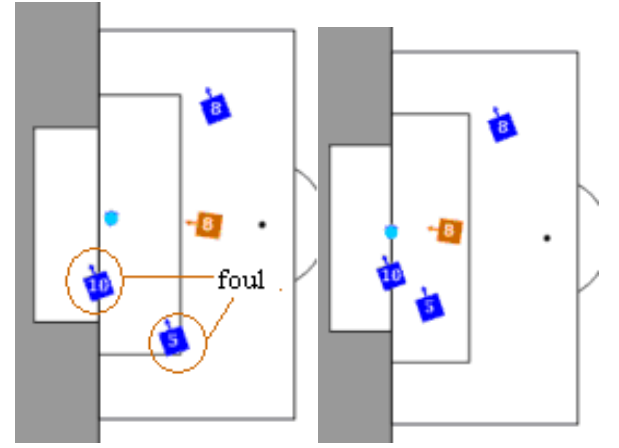

(a) The fifteenth period (b) The eighteenth period

Fig. 4. the situation of easily giving wrong judgment

\section{CONCLUSION}

This paper studies Conditional Event Algebra Theory,

combined with a good knowledge of the Bayesian network expression framework to draw a new assessment methods used in robot soccer simulation platform for smart referee. Experiments prove that the smart the referee has better intelligence, adaptability. However, deficiencies are still existed, such as computational complexity; accuracy is not high, needed after further efforts and improvements.

\section{REFERENCES}

[1] G. Philip and Calabrese. Calabrese toward a more natural expression of quantum logic with boolean fractions [J]. Space \& Naval Warfare Systems Center.

[2] I. R. Goodman and D. Bamber, "New applications of relational event algebra to fuzzy quantification and probabilistic reasoning [J]," Information Sciences, no.148, pp.87-96, 2002.

[3] Y. Deng, Q. Liu, and Wenkang Shi. A Review on Theory of Conditional Event Algebra [J]. Chinese Journal of Computers. 2003.06

[4] D. Bamber and I. R. Goodman, "deduction from conditional knowledge [J],” Soft Computing, vol.8, pp. 247-255, 2004.

[5] Y. Deng and Wenkang Shi, "Product Space Conditional Event Algebra and its Application [J].” Microelectronics \& Computer, 2001.04.

[6] D Bamber, "Extension of the concept of propositi-onal deduction from classical logic to probability an overview of probability-selection approaches [J],” Information Sciencens, vol. 131, pp.195-250, 2000 \& 2001.

[7] D. Bamber and I. R. Goodman, New uses of second order probability techniques in estimating critical probabilities in command \& control decision-making. Proceedings of the 2000 Command \& Control Research \& Technology Symposium, Naval Postgraduate School, Monterey, California, June 2000:26-28.

[8] I. R. Goodman and H. T. Nguyen, "Probability updating using second order probabilities and Conditional Event Algebra," Information Sciences, vol. 131, no. 3, pp. 295-347. 1999.

[9] J. Pearl, Probabilistic Reasoning in Intelligent Systems: Networks of Plausible Inference. San Mateo CA: Morgan Kaufman Publishers, 1988.

[10] Ajin Xu, Yong Yan, and Weiyi Liu. "Logic Inference in Bayesian Network Based on Conditional Event Algebra [J]." Computer Engineering and Applications. 2006.02.

[11] Yong Li and Weiyi Liu. A Method of Probabilist Reasoning on Bayesian Network [J]. Faculty of Information Engineering and Automation Kunming University of Science and Technology.2007.

[12] Yong Li and Wei-Yi Liu. Backward probabilistic logic reasoning algorithm for decision problem with Conditional Event ALgebra on Bayesian networks. [J]. Kunming University of Science and Technology.2008.07.
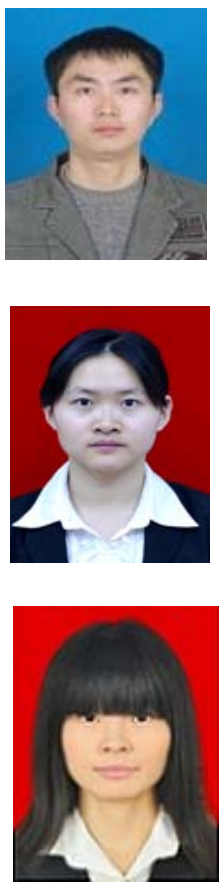

Zhu Shengguo was born in AnHui, China on June 1, 1985. He received the B.S.degree in information and computer science from Wuhan Institute of Technology, China in 2007. He is currently study the M.S. degree in pattern recognition and intelligent system from Huazhong University of Science \& Technology, Wuhan, China. His research interests include computer vision, intelligent algorithm and image processing.

Peng Li was born in Hubei, China on September 21, 1985. She received the B.S.degree in information and computer science from Wuhan Institute of Technology, China in 2007. She received the M.S. degree in computer science from Wuhan Institute of Technology, China in 2010. She is currently teaching computer science in Hubei Radio \& TV University. Her interests include intelligent algorithm and pattern recognition.

Zhan Lijuan was born in AnHui, China on June 20, 1989. She received the B.S.degree in biomedical engineering from Huazhong University of Science \& Technology, China in 2007. He is currently study the M.S. degree in pattern recognition and intelligent system from Huazhong University of Science \& Technology, Wuhan, China. His research interests include computer vision, intelligent algorithm and image processing. 